\section{MUCking about in IPF: identification of a novel goblet cell phenotype in pulmonary fibrosis}

\author{
Colin D Bingle
}

The airway epithelium, extending from the nasal cavity through the trachea and bronchial passages to the alveoli, is a complex tissue that performs a multitude of vital functions. This epithelium is generally considered to be composed of two contiguous divisions: the proximal conducting airway regions that serve as a conduit for inhaled air and the distal gas exchanging regions of the peripheral lung. This simple view does not adequately reflect the range of specialised activities necessary for normal lung functions. ${ }^{1}$ These include not only the key function of gas exchange between the respiratory and circulatory systems but also the synthesis and secretion of components of the extracellular lining of the lungs, uptake and metabolism of secreted products and xenobiotics, fluid and electrolyte balance, and protection and repair of the epithelium following injury. Accomplishing these various tasks requires an array of differentiated epithelial cells capable of sustaining these functions in distinct portions of the lungs. ${ }^{1}$ The surface epithelium of the cartilaginous conducting airways is a pseudostratified columnar epithelium composed of ciliated, non-ciliated, goblet, neuroendocrine and basal cell types. Interspersed between the cartilaginous rings of the larger airways are the submucosal glands (SMGs). Airway SMGs are microscopically similar to other minor mucosal glands that underlie the epithelium of the nasal passages and oral cavity and it is now clear that they are a major source of the secretions that coat the airway epithelium. As the epithelium extends further down into the lungs the structure becomes a less complex cuboidal epithelial layer containing a mixture of

Correspondence to Dr Colin D Bingle, Reader in Respiratory Cell and Molecular Biology, Academic Unit of Respiratory Medicine, Department of Infection and Immunity, University of Sheffield, LU111, Royal Hallamshire Hospital, Glossop Road, Sheffield S10 2JF, Sheffield, UK; c.d.bingle@sheffield.ac.uk ciliated and secretory cells until the epithelium in the alveolus is a mix of type I and type II pneumocytes intimately associated with the capillary bed.

There have been significant advances in the understanding of the molecular drivers of cellular commitment and differentiation of individual pulmonary cell types within the past 20 or so years. This area of investigation initially started through the study of cell-type restricted gene products, including the surfactant proteins (SP-A, B, $C$, and $D)^{2-5}$ and clara cell secretory protein/uteroglobin (secretoglobin1A1). ${ }^{6}$ Subsequently, this led to the identification of transcription factors, including members of the FOX (forkhead box) and homeodomain containing protein families, including HNF-3/FOXA, FOXJ1 and NKX2.1/TTF-1, which not only regulated the cell type specific expression of lung restricted genes in adult cells but were also implicated in the regulation of aspects of lung development, and cell commitment and differentiation of distinct epithelial cell populations. ${ }^{7}$ This area of pulmonary biology is continuing to generate a rich source of information on the processes that govern the establishment of the 'normal' epithelium, through studies of in vitro models of epithelial differentiation, such as the use of mucociliary differentiation of primary airway cells in air liquid interface cultures, ${ }^{8}$ as well as using genetargeted and modified animals for fate mapping and functional analysis. ${ }^{9}$

Clearly, a patent and fully functional airway epithelium is essential for pulmonary health and it is not surprising that epithelial injury and aberrant repair processes are associated with the majority of lung diseases. Well-characterised examples include post-transplant bronchiolitis obliterans syndrome, chronic obstructive pulmonary disease (COPD), cystic fibrosis and asthma. Some of these diseases (eg, asthma) result in phenotypic changes brought about by the process of epithelial mesenchymal transition ${ }^{1}$ while others result in changes to the epithelium through the process of mucus cell metaplasia (eg, COPD). ${ }^{10}$ Importantly, these two processes are not mutually exclusive between particular diseases. The paper in this issue of Thorax by Plantier et al (see page 651 ${ }^{11}$ provides a novel and important insight into the altered phenotype of the remodelled bronchiolised epithelium found in the lungs of patients with idiopathic pulmonary fibrosis (IPF), a disease in which the significance of mucous cell metaplasia has not been so actively studied. IPF is an irreversible and progressive lung disease with limited life expectancy after diagnosis. The incidence of IPF appears to be growing with around 4000 patients per year being diagnosed in the UK. ${ }^{12}$ This is in part due to better diagnostic procedures. The cause of the condition remains elusive but it has both familial and environmental associations. Recently, there has been much activity directed towards the identification of novel molecular markers of disease classification and progression but the utility of any such markers remains largely untested. ${ }^{13} 14$ While in many cases clinical and radiological features may allow a diagnosis, in many instances the pathologist remains key to the accurate diagnosis of IPF. On histopathological analysis, IPF lungs reveal what is known as the typical 'usual interstitial pneumonia' pattern of focal abnormalities including areas of epithelial hyperplasia, scarring with fibroblast foci and bronchiolisation of alveolar ducts, cysts and alveoli. Although much of the research on IPF has necessarily focused on the development of the characteristic fibrotic lesions, it is clear that major epithelial changes are found in this condition and indeed both the morphologically distinct processes are often present in different areas of the same lung. One of the most striking observations in this disease is the presence of a significant number of mucincontaining goblet cells within the bronchiolised epithelium. These cell types are normally absent from the distal lung epithelium. Mucin from these cells fills the honeycombed cysts. Although it seems likely that bronchiolar abnormalities are caused by changes in epithelial cell differentiation, the mechanisms governing this process are poorly understood. A number of studies in mouse models have begun to shed light on this process by showing that alveolar epithelial cell injury could be associated with the development of pulmonary fibrosis. ${ }^{15}$ Previous studies have implicated a number of molecular 
pathways that underpin this process in $\mathrm{IPF}^{16}{ }^{17}$ but the nature of the goblet cells found in this disease is not well characterised. Plantier et al identify a novel phenotype of these cells and show that they stain strongly for one of the major gel-forming mucins, MUC5B, but not with the related protein MUC5AC. Additionally, they show that these goblet cells are negative for two of the transcription factors previously associated with airway goblet cell development, FOXA3 and SPDEF. $^{18}$ Their data provide support for the growing concept that not all goblet cells are phenotypically identical. MUC5B and MUC5AC are major components of airway mucus and make a significant contribution to the abnormal viscous mucus seen in a number of chronic lung diseases. ${ }^{10}$ Normally, the major sites of MUC5B production are the SMGs whereas MUC5AC is the product of the goblet cell in the proximal airways. ${ }^{10}$ However, an increased SMG size and number has been associated with elevated mucus production in asthmatic and COPD airways. ${ }^{10}$ Ectopic MUC5B localisation has not previously been described in IPF and the data presented in this paper suggest that these cells have the characteristics of SMG goblet cells. To investigate these ectopic goblet cells further, the authors also studied the expression of neuregulins (NRGs) in these cases. NRGs are ligands for epidermal growth factor-related receptors and two alternatively spliced protein products of this protein, NRG1 $\alpha$ and NRG1 $\beta$ have been implicated in the production of goblet cells in vitro, with NRG1 $\alpha$ in particular being associated with the generation of MUC5B-positive goblet cells. ${ }^{19}$ NRGs have previously been shown to play roles in many aspects of biology including lung development and lung cancer, ${ }^{20}$ but until now their role in chronic lung disease has not been studied. The data in the present paper show that NRG1 $\alpha$ (but not NRG1 $\beta$ ) is localised to squamous epithelium adjacent to regions of MUC5B expressing goblet cells in the honeycombed lung. This is particularly interesting in view of the increased incidence of lung cancer in IPF patients. Both proteins were also shown to be present in airway SMGs, further supporting the view that NRG1 $\alpha$ is associated with MUC5B-positive mucous cell formation.
The fact that NRG1 $\alpha$ is associated with IPF was also shown by the increase of the protein (but again not of NRG1 $\beta$ ) in bronchoalveolar lavage fluid from a distinct group of patients with 'earlystage' IPF cases as compared with those having other interstitial lung diseases.

The data in this paper provide strong evidence that the mucous cell metapalsia seen in the bronchiolised epithelium of IPF cases is distinct from that seen in the airways of patients with COPD and asthma. It further suggests that a discrete set of molecular drivers are likely responsible for this phenotypic alteration. It remains to be seen if this is a genetic or environmental aspect of the disease. An increasing number of genetic associations have been described in $\mathrm{IPF}^{21}$ and recently interest has become focused on polymorphisms located in the gel-forming mucin gene locus that includes MUC5A/C and MUC5B, located on chromosome 11p15.5. ${ }^{22}$ Whether the alterations of MUC5B production seen in this paper are associated with genetic variants of the $M U C$ locus and if this altered expression is unique to MUC5B or is replicated with other SMG gene products, remains to be seen but suggests that this should be an area for intense research in the near future.

\section{Competing interests None.}

Provenance and peer review Commissioned; not externally peer reviewed.

Published Online First 17 April 2011

Thorax 2011;66:647-648.

doi:10.1136/thx.2011.161307

\section{REFERENCES}

1. Knight DA, Holgate ST. The airway epithelium: structural and functional properties in health and disease. Respirology 2003;8:432-46.

2. Bruno MD, Bohinski RJ, Huelsman KM, et al. Lung cell-specific expression of the murine surfactant protein A (SP-A) gene is mediated by interactions between the SP-A promoter and thyroid transcription factor-1. J Biol Chem 1995:270:6531-6.

3. Bohinski RJ, Huffman JA, Whitsett JA, et al. Cis-active elements controlling lung cell-specific expression of human pulmonary surfactant protein $B$ gene. J Biol Chem 1993;268:11160-6.

4. Glasser SW, Korfhagen TR, Wert SE, et al. Genetic element from human surfactant protein SP-C gene confers bronchiolar-alveolar cell specificity in transgenic mice. Am J Physiol 1991;261:L349-56.

5. Rust K, Bingle L, Mariencheck W, et al. Characterization of the human surfactant protein $D$ promoter: transcriptional regulation of SP-D gene expression by glucocorticoids. Am J Respir Cell Mol Biol 1996:14:121-30.
6. Bingle CD, Gitlin JD. Identification of hepatocyte nuclear factor-3 binding sites in the Clara cell secretory protein gene. Biochem $J$ 1993;295:227-32.

7. Hackett BP, Bingle CD, Gitlin JD. Mechanisms of gene expression and cell fate determination in the developing pulmonary epithelium. Annu Rev Physiol 1996:58:51-71.

8. Ross AJ, Dailey LA, Brighton LE, et al. Transcriptional profiling of mucociliary differentiation in human airway epithelial cells. Am J Respir Cell Mol Biol 2007;37:169-85.

9. Rawlins EL, Okubo T, Que J, et al. Epithelial stem/ progenitor cells in lung postnatal growth, maintenance, and repair. Cold Spring Harb Symp Quant Biol 2008;73:291-5.

10. Curran DR, Cohn L. Advances in mucous cell metaplasia: a plug for mucus as a therapeutic focus in chronic airway disease. Am J Respir Cell Mol Biol 2010;42:268-75

11. Plantier L, Crestani B, Wert SE, et al. Ectopic respiratory epithelial cell differentiation in bronchiolised distal airspaces in idiopathic pulmonary fibrosis. Thorax 2011;66:651-7.

12. Gribbin J, Hubbard RB, Le Jeune I, et al. Incidence and mortality of idiopathic pulmonary fibrosis and sarcoidosis in the UK. Thorax 2006;61:980-5.

13. Boon K, Bailey NW, Yang J, et al. Molecular phenotypes distinguish patients with relatively stable from progressive idiopathic pulmonary fibrosis (IPF). PLoS One 2009; 4:e5134.

14. Konishi K, Gibson KF, Lindell KO, et al. Gene expression profiles of acute exacerbations of idiopathic pulmonary fibrosis. Am J Respir Crit Care Med 2009;180:167-75.

15. Glasser SW, Detmer EA, Ikegami M, et al Pneumonitis and emphysema in SP-C gene targeted mice. J Biol Chem 2003;278: 14291-8.

16. Chilosi M, Poletti V, Murer B, et al. Abnormal re-epithelialization and lung remodeling in idiopathic pulmonary fibrosis: the role of deltaN-p63. Lab Invest 2002;82:1335-45

17. Chilosi M, Poletti V, Zamò A, et al. Aberrant Wnt/ beta-catenin pathway activation in idiopathic pulmonary fibrosis. Am J Pathol 2003; 162:1495-502.

18. Chen G, Korfhagen TR, Xu Y, et al. SPDEF is required for mouse pulmonary goblet cell differentiation and regulates a network of genes associated with mucus production. J Clin Invest 2009:119:2914-24.

19. Kettle R, Simmons J, Schindler F, et al. Regulation of neuregulin beta-induced MUC5AC and MUC5B expression in human airway epithelium. Am J Respir Cell Mol Biol 2010;42:472-81.

20. Esper RM, Pankonin MS, Loeb JA. Neuregulins: versatile growth and differentiation factors in nervous system development and human disease. Brain Res Rev 2006;51:161-75.

21. Lawson WE, Loyd JE. Will the genes responsible for familial pulmonary fibrosis provide clues to the pathogenesis of IPF? Am J Respir Crit Care Med 2010;182:1342-3.

22. Seibold MA, Lin J, Kervitsky D, et al. The concentration of MUC5AC is higher in the air space of idiopathic pulmonary fibrosis (IPF) subjects and is associated with a disease susceptibility variant (Ala497Val) in MUC5AC. Am J Respir Crit Care Med 2010:181:A2493. 